\title{
Development and evaluation of an early death risk prediction model after acute type $A$ aortic dissection
}

\author{
Yuhui Zhang ${ }^{1,2 \#}$, Tongyun Chen ${ }^{2 \#}$, Qingliang Chen $^{2}$, Hou Min ${ }^{3}$, Jiang Nan ${ }^{2}$, Zhigang Guo ${ }^{2}$ \\ ${ }^{1}$ Clinial College of Chest, Tianjin Medical University, Tianjin, China; ${ }^{2}$ Department of Cardiovascular Surgery, Tianjin Chest Hospital, Tianjin, \\ China; ${ }^{3}$ Clinical Laboratory, Tianjin Chest Hospital, Tianjin, China \\ Contributions: (I) Conception and design: Y Zhang, Q Chen; (II) Administrative support: Z Guo, J Nan; (III) Provision of study materials or patients: \\ Y Zhang, Q Chen, J Nan, T Chen; (IV) Collection and assembly of data: Y Zhang, T Chen, H Min; (V) Data analysis and interpretation: Y Zhang, \\ T Chen; (VI) Manuscript writing: All authors; (VII) Final approval of manuscript: All authors. \\ \#These authors contributed equally to this work. \\ Correspondence to: Prof. Qingliang Chen, MD; Prof. Zhigang Guo, MD. Department of Cardiovascular Surgery, Tianjin Chest Hospital, South \\ Taierzhuang Road No. 261, Tianjin 300222, China. Email: welych@163.com; zhigangguo@vip.163.com.
}

Background: The purpose of the study was to assess the relationship between preoperative laboratory examination, clinical imaging data, and postoperative death of patients with acute type A aortic dissection (ATAAD) and to establish a prediction model of hospital death risk after the operation.

Methods: A total of 224 cases of acute Standford A aortic dissection were treated by total arch replacement using a tetrafurcate graft with stented elephant trunk implantation in Tianjin Chest Hospital. Based on preoperative laboratory examination and clinical imaging data of patients with ATAAD, the independent risk factors of postoperative hospital death were obtained using logistic analysis, and a risk prediction model of postoperative hospital death was developed.

Results: Independent risk factors of postoperative death in patients with ATAAD were: body mass index (BMI), preoperative neutrophil to lymphocyte ratio (NLR), mean platelet volume (MPV), creatinine (Cr), D-dimer, high-sensitive cardiac troponin T (hs-CTnT), apolipoprotein A1, left subclavian artery involvement, and iliac artery involvement. The regression equation of postoperative death risk was: logitP1 $=-9.584+1.060 \times \mathrm{NLR}+1.586 \times \mathrm{MPV}+1.009 \times \mathrm{Cr}+1.067 \times \mathrm{D}$-dimer $+2.023 \times$ hs-CTnT; the regression equation of postoperative death risk was: $\operatorname{logitP} 2=-3.296+3.242 \times$ left subclavian artery involved +4.564 $\times$ iliac artery involved; the regression equation of postoperative death risk was: logitP $3=-12.864+1.149 \times$ $\mathrm{BMI}+4.731 \times$ left subclavian artery involved $+4.150 \times$ iliac artery involved $+1.064 \times \mathrm{NLR}+1.011 \times \mathrm{Cr}+$ $1.084 \times$ D-dimer $+2.242 \times$ hs-CTnT $+3.233 \times$ apolipoprotein A1.

Conclusions: BMI, NLR, MPV, Cr, D-dimer, hs-CTnT, apolipoprotein A1, left subclavian artery involvement and iliac artery involvement can affect the hospital mortality rate of aortic dissection undergoing Sun's operation to varying degrees, which may be helpful to guide the design of the perioperative treatment strategy.

Keywords: Acute type A aortic dissection (ATAAD); risk factors of surgical treatment; prediction of death; postoperative hospital death

Submitted Jul 16, 2021. Accepted for publication Sep 02, 2021.

doi: $10.21037 / \mathrm{atm}-21-4063$

View this article at: https://dx.doi.org/10.21037/atm-21-4063 


\section{Introduction}

Acute type A aortic dissection (ATAAD) is a dangerous and fatal cardiovascular disease. The incidence rate is 0.005 $0.030 \%$, the onset is urgent, the disease progresses rapid, and the mortality rate high. Related studies show that the mortality rate of ATAAD patients is $1-2 \%$ per hour within 48 hours after onset, $70 \%$ within 1 week and $80 \%$ within 2 weeks. For ATAAD, total aortic arch replacement under deep hypothermic circulatory arrest is the most important treatment. Even with the improvements of surgical technique and perioperative management, the mortality rate after surgical treatment is still as high as $10 \%$ to $30 \%$ (1). Old age, preoperative organ perfusion, cardiac tamponade, hypotension, postoperative stroke, CRRT treatment, low cardiac output syndrome, and multiple organ failure are all high risk factors for early death. The main reasons for the need for reoperation were the formation of residual distal aortic aneurysm, the stenosis and poor perfusion of abdominal artery branches, and the progressive aortic regurgitation. In 2002, Professor Sun Lizhong, China, put forward an improved surgical technique of replacing the whole aortic arch with four bifurcated artificial blood vessels and implanting new stent artificial blood vessels into the descending aorta to treat complex aortic dissection. It can significantly reduce the postoperative mortality and the reoperation rate. This technique is also called Sun's procedure, and it has achieved good therapeutic results for ATAAD (2).

It is very important for the prognosis of patients with aortic dissection to see a doctor to diagnose, accurately evaluate the condition and implement reasonable and effective treatment in a timely manner. Previous studies $(3,4)$ have made it clear that patients with severe cerebral vascular hypoperfusion, abdominal organ hypoperfusion, and coronary artery hypoperfusion have high postoperative complication risk and mortality rates, but at present, the risks affecting postoperative death are still rarely put forward. Preoperative clinical manifestations, examinations and imaging data of patients are not only conducive to clear diagnosis but also can reflect the severity of the patient's illness, which in turn has a positive predictive value for the prognosis. This study obtained basic information of patients before the operation, including laboratory and clinical imaging data, and quantitatively evaluated and predicted the risk of death following surgical treatment. The latter is conducive to guiding the timely implementation of effective clinical treatment and improving the prognosis. We present the following article in accordance with the STARD reporting checklist (available at https://dx.doi.org/10.21037/ atm-21-4063).

\section{Methods}

In this study, perioperative clinical data of patients with ATAAD at Tianjin Chest Hospital were collected, and the correlations between preoperative data and early postoperative death were analyzed. Independent risk factors related to postoperative death were analyzed using logistic regression, and a prediction model of early postoperative death was developed. All procedures performed in this study involving human participants were in accordance with the Declaration of Helsinki (as revised in 2013). This study is a single-center retrospective study, which has been approved by the Ethics Committee of Tianjin Chest Hospital. Individual consent for this retrospective analysis was waived.

\section{General information}

Clinical information of patients with ATAAD who were hospitalized in Tianjin Chest Hospital from January 1, 2017, to January 1, 2020, was collected. All patients were diagnosed with ATAAD using computed tomography angiography (CTA), which has $100 \%$ sensitivity and $99 \%$ specificity for the diagnosis, and color Doppler echocardiography before the operation. After admission, blood samples were obtained from all patients through the cubital vein for initial testing to assess biochemistry, probrain natriuretic peptide, high-sensitive cardiac troponin $\mathrm{T}$ (hs-CTnT), D-dimer, and other laboratory values. Routine blood tests were conducted using SYSMEX XN9000 (Sysmex, Kobe, Japan). Biochemical tests were conducted using COBAS C701 (Roche Diagnostics, Indianapolis, IN, USA). Information on clinical manifestation, past medical history, laboratory examination data, CTA imaging examination, cardiac color Doppler ultrasound data, and perioperative data were collected (see Tables 1,2).

The following patients were excluded: those with a delay of the operation of 14 days or more; those with traumatic, iatrogenic aortic dissection, re-aortic dissection, aortic aneurysm, Marfan syndrome, hematological diseases, new cerebral infarction and cerebral hemorrhage before operation, or acute myocardial infarction $>6$ hours with hemodynamic instability as were patients, who were dialysis-dependent. A total of 224 patients were included. Among them, there were 172 males (76.8\%) and 52 females 
Table 1 Preoperation testing data of 224 type A aortic dissection patients (mean \pm SD)

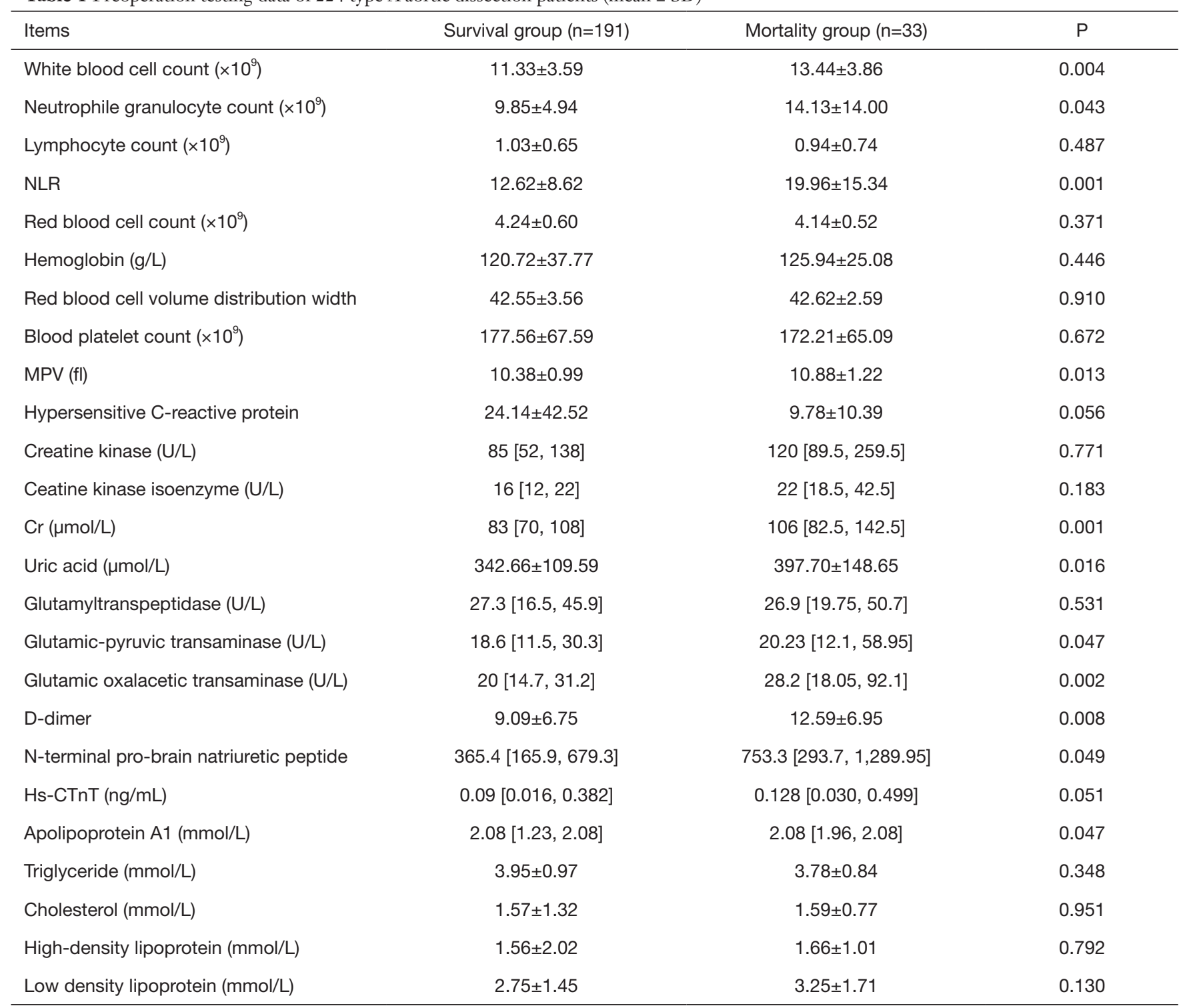

$\mathrm{SD}$, standard deviation; NLR, neutrophil to lymphocyte ratio; MPV, mean platelet volume; Cr, creatinine; hs-CTnT, high-sensitive cardiac troponin $\mathrm{T}$.

$(23.2 \%)$ with an age of $(52.76 \pm 11.73)$ years.

After admission, all patients were treated with individualized interventions, with systolic blood pressure controlled at $110-120 \mathrm{mmHg}$, heart rate $\leq 60$ beats/min, analgesia, and sedation. Observation endpoints of selected patients were: Postoperative survival, vital signs were stable after surgical treatment, patient survived and met the criteria for being discharged from hospital; hospital death after operation, including death caused by various complications; and use of mechanical auxiliary devices for continuous extracorporeal life support, with no improvement in independent life status, and treatment was ceased.

Operation: the operation was performed by an operating team comprising the same members. Intravenous anesthesia, right internal jugular vein catheterization, left radial artery and left dorsalis pedis artery catheterization for pressure measurement were performed; intraoperative cardiopulmonary bypass was established through femoral artery intubation and superior and inferior vena cava intubation; and a left atrial drainage tube was inserted 
Table 2 Preoperative clinical imaging data of 224 type A aortic dissection patients [mean \pm SD/n (\%)]

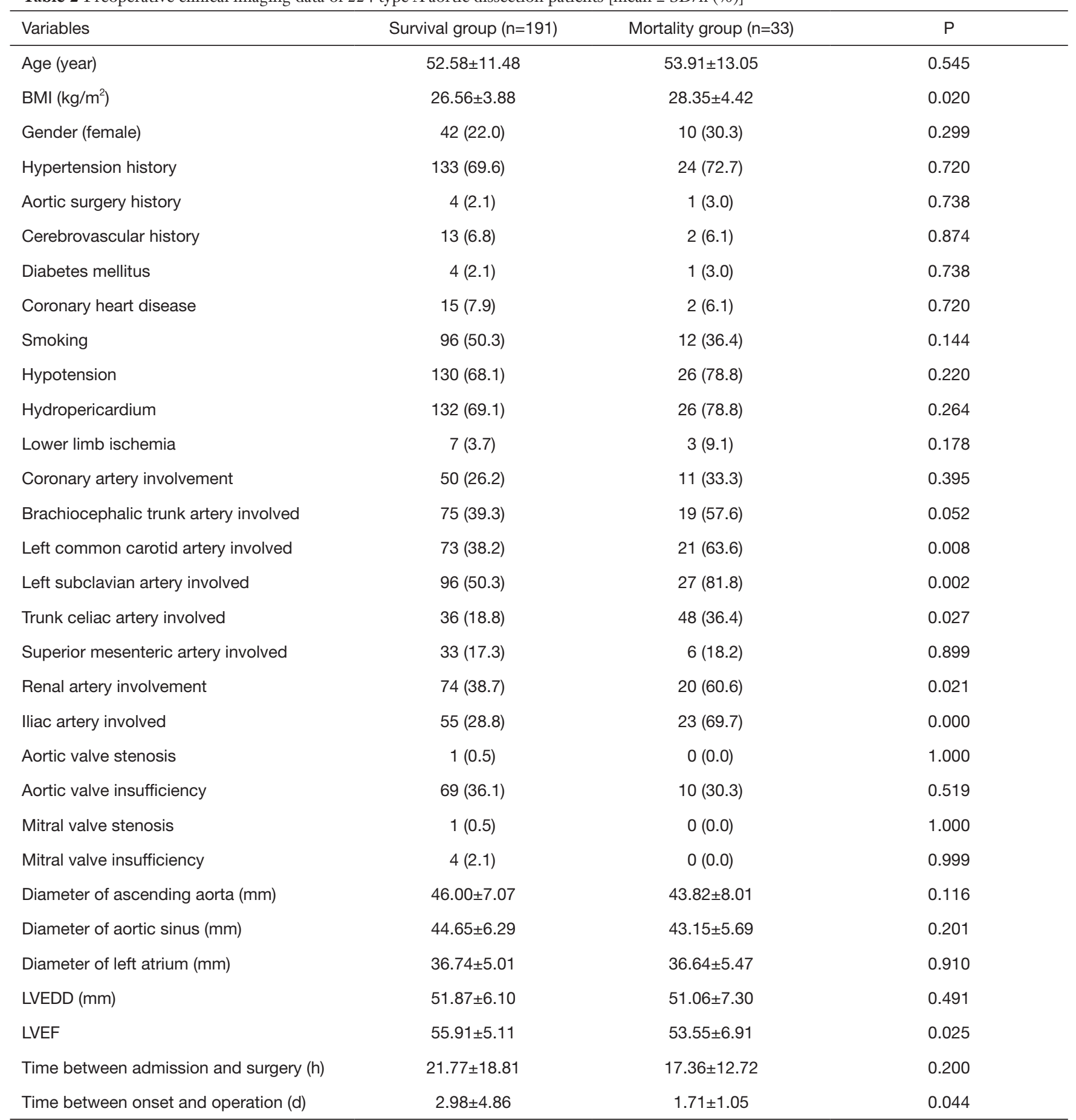

SD, standard deviation; BMI, body mass index; LVEDD, left ventricular end-diastolic diameter; LVEF, left ventricular ejection fraction.

into the right superior pulmonary vein. The nasopharynx temperature was decreased to $32{ }^{\circ} \mathrm{C}$ to block the ascending aorta, and the aortic root was perfused with histidine- tryptophan-ketoglutarate (HTK) cardioplegia. According to the specific pathological changes of the aortic root, Bentall, Wheat and sinus shaping were selected for root repair. The 
nasopharyngeal temperature was decreased to $28{ }^{\circ} \mathrm{C}$, the left common carotid artery and innominate artery were intubated anterograde, and cerebral perfusion stopped the circulation. The aortic arch was opened, the brachiocephalic artery was crossed, the left common carotid artery and the left subclavian artery were also crossed, the aortic arch was disconnected at the proximal end of the left subclavian artery and the proximal end of the left subclavian artery was closed. A Cronus stent-graft (MicroPort, Shanghai, China) was implanted into the distal end of the descending thoracic aorta, and the distal end of the trunk was anastomosed with the proximal end of the descending thoracic aorta using Hemashield Platinum (Getinge, Gothenburg, Sweden). Anastomosis of the proximal end of the artificial blood vessel trunk with the ascending aorta was performed, the temperature was increased again, the ascending aorta was opened, and the heart beat again; the anastomosis of the left subclavian artery was continued, using the left common carotid artery, brachiocephalic artery, and artificial blood vessel, then the left common carotid artery and brachiocephalic artery intubation were pulled out, the circulation was adjusted, the cardiopulmonary bypass was stopped at a nasopharyngeal temperature of $35^{\circ} \mathrm{C}$, bleeding was stopped and the chest was closed. Patients with severe cardiac, pulmonary and renal insufficiency following the operation should be treated using extracorporeal membrane oxygenation (ECMO) and continuous renal replacement therapy (CRRT).

\section{Statistical methods}

Continuous measurement data were analyzed using the Shapiro-Wilk test, and the results of the normal distribution are reported using mean \pm standard deviation (SD). Independent-samples $t$-tests were used for comparisons between the two groups. Data of non-normally distributed data are reported using the median $(\mathrm{M})$ and interquartile range (P25, $\mathrm{P} 75)$, and the comparisons between the two groups were conducted using rank-sum test of two independent samples. All the numerical data are reported using frequencies and percentage. For comparisons between the two groups, the $\gamma$-test or Fisher's exact test was used, and the difference was considered statistically significant with $\mathrm{P}<0.05$. Odds ratios (ORs) including $95 \%$ confidence intervals (CIs) are reported for logistic regression analysis. The selected preoperative variables were analyzed by using binary logistic regression, and $\mathrm{P}<0.1$ was chosen to select death risk-related factors for inclusion in regression. Then, the stepwise backward likelihood ratio method was used to perform multivariate logistic regression analysis, whereby independent risk factors related to postoperative death were identified, the regression equation was obtained, and the death risk model was developed. The predicted probability was analyzed using receiver operating characteristic (ROC) curves, and the area under the ROC curve (AUC) was obtained. An AUC $>0.7$ indicates good discrimination and clinical applicability. The model calibration degree was evaluated using the Hosmer-Lemeshow test (H-L goodness-of-fit test), and the model calibration degree was considered better with $\mathrm{P}>0.05$. Subjects missing more than $10 \%$ of their data were excluded from the group. All data were statistically analyzed using SPSS version 25.0 software.

\section{Results}

\section{Preoperative demographic characteristics}

A total of 224 patients were enrolled in this study, all of whom had ATAAD, including 172 males $(76.8 \%)$ and 52 females $(23.2 \%)$, aged $52.76 \pm 11.73$ years. Ten cases (4.5\%) had lower limb ischemia symptoms before the operation. After admission, venous blood samples were taken for routine blood tests, biochemical tests, and probrain natriuretic peptide and hs-CTnT tests. The clinical imaging data, laboratory examination data, and surgical data of patients were collected and summarized (see test data, Table 1; clinical imaging data, Table 2).

\section{Intraoperative characteristics}

Cardiopulmonary bypass was established using intravenous anesthesia, femoral artery intubation, anterograde cerebral perfusion of brachiocephalic artery and left common carotid artery and superior and inferior vena cava intubation. Total arch replacement with Hemashield Platinum and Cronus stent-graft implantation in the descending aorta were the basic surgical strategies. Sixty-seven cases (29.9\%) underwent Bentall operation, 7 cases $(0.1 \%)$ underwent Wheat operation, and 150 cases (70\%) underwent ascending aorta replacement. The duration of cardiopulmonary bypass operations was $166.84 \pm 44.38 \mathrm{~min}$ in overall patients, $160.57 \pm 30.30 \mathrm{~min}$ in the survival group and 203.12 \pm $81.73 \mathrm{~min}$ in the death group. Aortic occlusion time was $97.10 \pm 23.47 \mathrm{~min}$ overall, $96.01 \pm 22.01 \mathrm{~min}$ in the survival group and $103.42 \pm 30.22 \mathrm{~min}$ in the death group. The circulatory arrest time of deep hypothermia was $12.76 \pm$ 
Table 3 Results of multiple logistic regression analysis

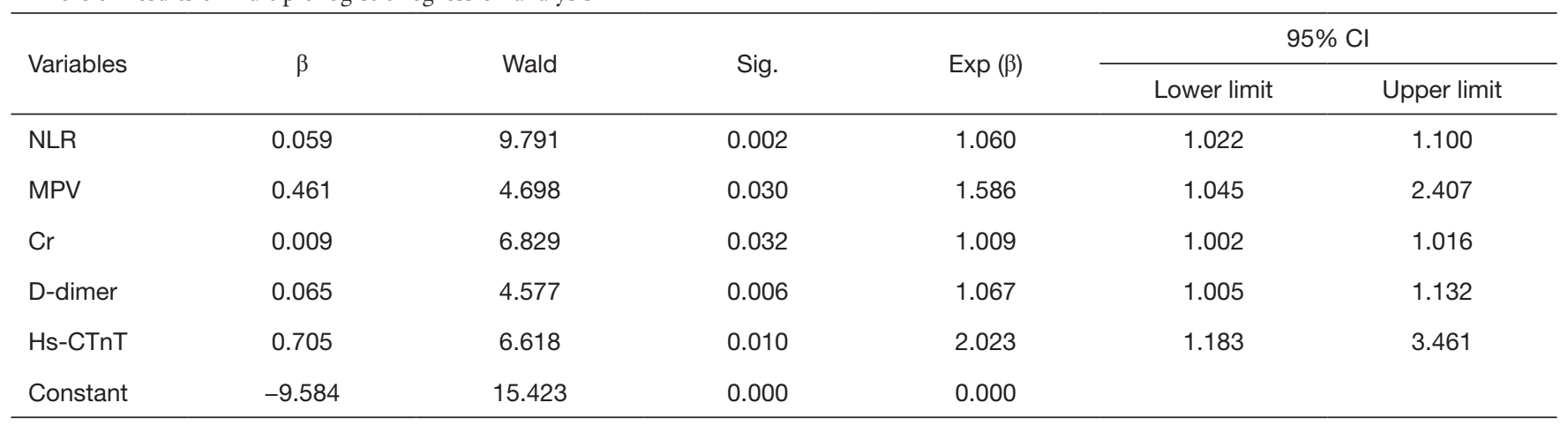

Sig., significance; $\mathrm{Cl}$, confidence interval; NLR, neutrophil to lymphocyte ratio; MPV, mean platelet volume; Cr, creatinine; hs-CTnT, highsensitive cardiac troponin $\mathrm{T}$.

$7.60 \mathrm{~min}$ overall, $13.05 \pm 7.61 \mathrm{~min}$ in the survival group and $11.03 \pm 7.40 \mathrm{~min}$ in the death group. Intraoperative blood loss was $1,340.15 \pm 636.40 \mathrm{~mL}$ overall, $1,313.87 \pm 570.58 \mathrm{~mL}$ in the survival group and $1,492.24 \pm 928.10 \mathrm{~mL}$ the death group.

\section{Surgical results}

In all, 191 cases (85.3\%) survived, and 33 cases (14.7\%) died after the operation. In the death group, there were 15 cases $(45.5 \%)$ with permanent cerebral infarction, 19 cases (57.6\%) with renal failure with CRRT, 12 cases (36.4\%) with respiratory failure with mechanical ventilation time longer than 72 hours, and 12 cases (36.4\%) with heart failure with cardiotonic drugs and mechanical device circulation assistance. Infection was the initial cause of multiple organ failure and infection.

\section{Development of death prediction model}

\section{Laboratory inspection data model (model 1)}

Binary logistic regression analysis was carried out to identify the risk factors related to surgical death. White blood cell count $(\mathrm{P}=0.004)$, neutrophil count $(\mathrm{P}=0.043)$, neutrophil to lymphocyte ratio (NLR; $\mathrm{P}=0.001$ ), mean platelet volume (MPV; $\mathrm{P}=0.013$ ), high-sensitivity $\mathrm{C}$-reactive protein $(\mathrm{P}=0.056)$, creatinine $(\mathrm{Cr} ; \mathrm{P}=0.001)$, uric acid $(\mathrm{P}=0.016), \mathrm{D}$-dimer $(\mathrm{P}=0.008)$, pro-brain natriuretic peptide $(\mathrm{P}=0.049)$, hs-CTnT $(\mathrm{P}=0.051)$, and apolipoprotein A1 $(\mathrm{P}=0.047)$ were associated with postoperative death. The NLR [P=0.002, OR: 1.060 (95\% CI: 1.022, 1.100)] and the average platelet volume $[\mathrm{P}=0.030$, OR: $1.586(95 \%$ CI: $1.045,2.407)]$ were obtained using stepwise backward likelihood analogy, while D-dimer $[\mathrm{P}=0.006$, OR: 1.067 (95\% CI: 1.005, 1.132)] and hs-CTnT [P=0.010, OR: 2.023 (95\% CI: 1.183, 3.461)] were still significantly related to postoperative death (Table 3).

The regression equation was as follows: $\operatorname{logitP} 1=-9.584$ $+1.060 \times \mathrm{NLR}+1.586 \times \mathrm{MPV}+1.009 \times \mathrm{Cr}+1.067 \times$ D-dimer $+2.023 \times$ hs-CTnT. With this regression formula, we predicted the probability $\mathrm{P} 1$ of death after the operation based on laboratory data by establishing the ROC curve (Figure 1) of the predicted probability P1. The AUC under the curve is 0.794 (95\% CI: $0.703,0.884)$. The HosmerLemeshow test was $\chi^{2}=3.073, \mathrm{P}>0.05$. The model has high discrimination and a calibration degree with good clinical applicability.

\section{Clinical imaging data model (model 2)}

Clinical imaging data were analyzed using binary logistic regression to identify the risk factors related to death. These were: body mass index (BMI; $\mathrm{P}=0.020)$, brachiocephalic trunk artery $(\mathrm{P}=0.052)$, left common carotid artery $(\mathrm{P}=0.008)$, left subclavian artery $(\mathrm{P}=0.002)$, abdominal trunk artery $(\mathrm{P}=0.027)$, renal artery $(\mathrm{P}=0.021)$, and iliac artery $(\mathrm{P}<0.001)$. Subsequent multivariate logistic regression analysis showed that the left subclavian artery $[\mathrm{P}=0.017$, OR: 3.242 (95\% CI: 1.239, 8.483)] and iliac artery [ $\mathrm{P}<0.001$, OR: 4.564 (95\% CI: 1.998, 10.424)] were independently related to postoperative death (Table 4).

The regression equation obtained was as follows: $\operatorname{logit} 2=-3.296+3.242 \times$ left subclavian artery involved $+4.564 \times$ iliac artery involved. In this way, the prediction probability $\mathrm{P} 2$ of the clinical image regarding postoperative death risk was obtained, and the ROC curve (Figure 2) of prediction probability $\mathrm{P} 2$ was established. The AUC under 


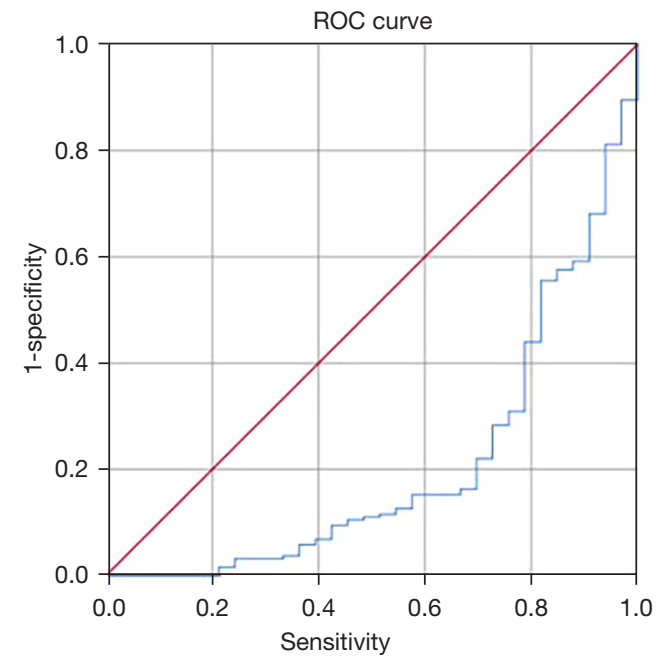

Figure 1 The ROC curve of P1, which is the multivariate regression result of the test data. The AUC is $0.794,95 \% \mathrm{CI}$ : $(0.703,0.884)$. It indicates that the prediction model has a good degree of differentiation. Regression equation: $\operatorname{logitP} 1=-9.584$ $+1.060 \times \mathrm{NLR}+1.586 \times \mathrm{MPV}+1.009 \times \mathrm{Cr}+1.067 \times \mathrm{D}$-dimer $+2.023 \times$ hs-CTnT. ROC, receiver operating characteristic; AUC, area under the ROC curve; CI, confidence interval; NLR, neutrophil to lymphocyte ratio; MPV, mean platelet volume; $\mathrm{Cr}$, creatinine; hs-c TnT, high-sensitive cardiac troponin T.

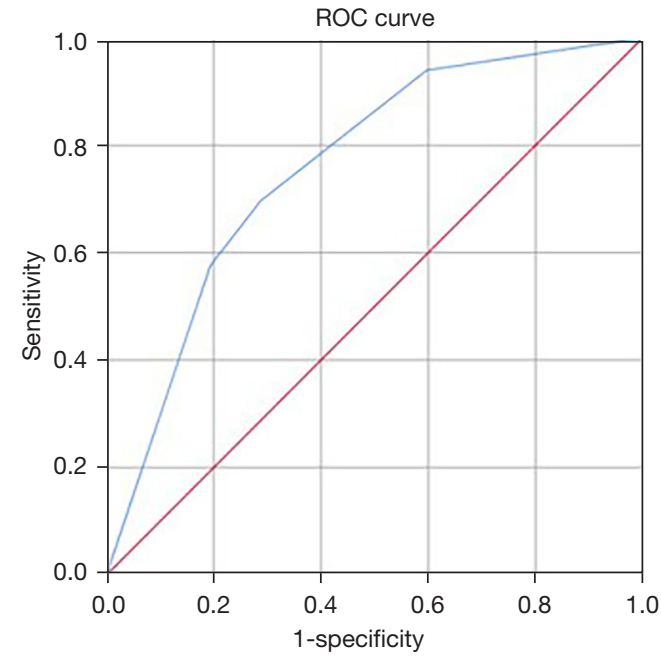

Figure 2 The ROC curve of P2, which is the multivariate regression result of the clinical imaging data. The AUC is 0.759 , $95 \%$ CI: $(0.677,0.841)$. It indicates that the prediction model has a good degree of differentiation. Regression equation: logitP2 $=-3.296+3.242 \times$ left subclavian artery involved $+4.564 \times$ iliac artery involved. ROC, receiver operating characteristic; AUC, area under the ROC curve; CI, confidence interval.

Table 4 Multivariate logistic regression analysis results of clinical imaging data

\begin{tabular}{|c|c|c|c|c|c|c|}
\hline \multirow{2}{*}{ Variables } & \multirow{2}{*}{$\beta$} & \multirow{2}{*}{ Wald } & \multirow{2}{*}{ Sig. } & \multirow{2}{*}{$\operatorname{Exp}(\beta)$} & \multicolumn{2}{|c|}{$95 \% \mathrm{Cl}$} \\
\hline & & & & & Lower limit & Upper limit \\
\hline Left subclavian artery involved & 1.176 & 5.746 & 0.017 & 3.242 & 1.239 & 8.483 \\
\hline Iliac artery involved & 1.518 & 12.982 & 0.000 & 4.564 & 1.998 & 10.424 \\
\hline Constant & 3.296 & 47.607 & 0.000 & 0.037 & & \\
\hline
\end{tabular}

Sig., significance; $\mathrm{Cl}$, confidence interval.

the curve was 0.759 (95\% CI: $0.677,0.841)$. The results of a Hosmer-Lemeshow test showed that the $\chi^{2}$ value was $0.652, \mathrm{P}>0.05$, which indicated that the model had a good fit, high discrimination and calibration, and good clinical applicability.

\section{Laboratory test data and clinical image data model (model 3)}

Univariate regression analysis was carried out on laboratory data and clinical imaging data, with $\mathrm{P}<0.1$ used to select variables for inclusion in multivariate regression analysis. The results were as follows: NLR [P=0.009, OR: 1.064
(95\% CI: 1.016, 1.114)], Cr [P=0.009, OR: 1.011 (95\% CI: $1.003,1.019)]$, D-dimer [P=0.022, OR: $1.084(95 \%$ CI: $1.012,1.162)]$, hs-CTnT $[\mathrm{P}=0.006$, OR: $2.242(95 \%$ CI: $1.258,3.998)]$, apolipoprotein $\mathrm{A} 1[\mathrm{P}=0.043$, OR: 3.233 (95\% CI: 1.038, 10.070)], BMI [P=0.016, OR: 1.149 (95\% CI: 1.027, 1.286)], subclavian artery $[\mathrm{P}=0.009$, OR: 4.731 (95\% CI: $1.464,15.282)]$, iliac artery $[\mathrm{P}=0.005$, OR: 4.150 (95\% CI: 1.551, 11.102)] (Table 5). The regression equation was $\operatorname{logitP} 3=-12.864+1.149 \times \mathrm{BMI}+4.731 \times$ left subclavian artery involved $+4.150 \times$ iliac artery involved $+1.064 \times \mathrm{NLR}+1.011 \times \mathrm{Cr}+1.084 \times \mathrm{D}$-dimer $+2.242 \times$ hs-CTnT $+3.233 \times$ apolipoprotein A1. According to this 
Table 5 Multivariate logistic regression analysis results of test and clinical imaging data

\begin{tabular}{|c|c|c|c|c|c|c|}
\hline Variables & $\beta$ & Wald & Sig. & $\operatorname{Exp}(\beta)$ & \multicolumn{2}{|c|}{$95 \% \mathrm{Cl}$} \\
\hline NLR & 0.062 & 6.859 & 0.009 & 1.064 & 1.016 & 1.114 \\
\hline $\mathrm{Cr}$ & 0.011 & 6.857 & 0.009 & 1.011 & 1.003 & 1.019 \\
\hline D-dimer & 0.081 & 5.251 & 0.022 & 1.084 & 1.012 & 1.162 \\
\hline Apolipoprotein A1 & 1.173 & 4.096 & 0.043 & 3.233 & 1.038 & 10.070 \\
\hline BMI & 0.139 & 5.846 & 0.016 & 1.149 & 1.027 & 1.286 \\
\hline Left subclavian artery involved & 1.554 & 6.747 & 0.009 & 4.731 & 1.464 & 15.282 \\
\hline Iliac artery involved & 1.423 & 8.031 & 0.005 & 4.150 & 1.551 & 11.102 \\
\hline
\end{tabular}

Sig., significance; Cl, confidence interval; NLR, neutrophil to lymphocyte ratio; Cr, creatinine; hs-CTnT, high-sensitive cardiac troponin T; BMI, body mass index.

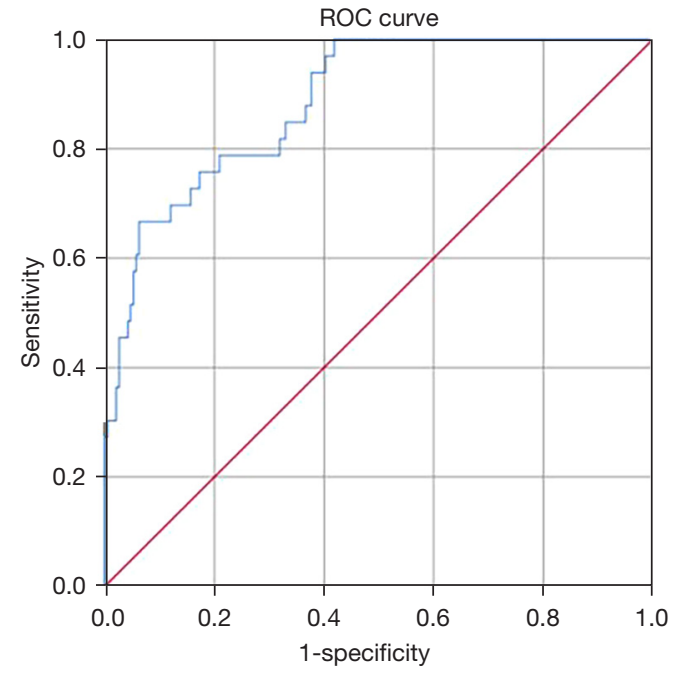

Figure 3 The ROC curve of P3, which is the multivariate regression result of the clinical imaging and testing data. The AUC is $0.886,95 \% \mathrm{CI}:(0.832,0.941)$. It indicates that the prediction model has a good discrimination and good practical applicability. The AUC of model 3 is greater than the model 1 (AUC $=0.794)$ and model 2 (AUC $=0.759)$. It indicates that model 3's predictive effect is better than that of the two other models. Regression equation: $\operatorname{logitP} 3=-12.864+1.149 \times \mathrm{BMI}+4.731 \times$ left subclavian artery involved $+4.150 \times$ iliac artery involved +1.064 $\times \mathrm{NLR}+1.011 \times \mathrm{Cr}+1.084 \times \mathrm{D}$-dimer $+2.242 \times$ hs-CTnT +3.233 $\times$ apolipoprotein A1. ROC, receiver operating characteristic; AUC, area under the ROC curve; CI, confidence interval; BMI, body mass index; NLR, neutrophil to lymphocyte ratio; Cr, creatinine; hs-CTnT, high-sensitive cardiac troponin T. formula, the predicted probability $\mathrm{P} 3$ was obtained, the ROC curve (Figure 3) of the predicted probability P3 was established, and the resulting AUC was 0.886 (95\% CI: $0.832,0.941)$. The Hosmer-Lemeshow test results of this model were $\chi^{2}=12.043, \mathrm{P}>0.05$, which shows that this model has a good fit, and high discrimination and calibration. The AUC of model 3 is greater than that of model 1 and 2 , which indicates that the former is better than the latter two in evaluating the risk of postoperative death. These findings suggest that patient data and clinical images should be considered comprehensively in clinical practice as far as possible, and that such data and images are helpful in improving the analysis and diagnosis of disease conditions and in predicting the prognosis and in particular possible death after evaluation.

\section{Discussion}

Although the incidence of ATAAD is low, it is dangerous, progresses rapidly and has a high mortality rate. Accurate preoperative assessment of the severity of the disease and timely formulation of a reasonable treatment plan can effectively improve the prognosis and reduce the mortality rate. In this study, we established a simple, effective, accurate, and practical model for predicting the death risk of type A aortic dissection surgery, which can provide guidance for the timely and effective treatment of such patients.

Aortic dissection is closely related to inflammation, which plays an important role in the occurrence, development, 
and prognosis of aortic dissection. After the occurrence of aortic dissection, macrophages in the media are activated, releasing metalloproteinases and acetate ductile cells, resulting in matrix degradation and cardiovascular formation. Inflammation can affect the degradation of the aortic middle layer and the remodeling of the arterial wall, which leads to weakness of the aortic wall and increases the probability of rupture. White blood cells and several subtypes are sensitive inflammatory markers, and the increase in the number of white blood cells reflects the inflammation and injury of dissected aortic vessel wall. Related studies show that white blood cell count, neutrophil count, and granulocyte/lymphocyte ratio have predictive value not only for the prognosis of cardiovascular disease, but also for the prognosis of acute aortic dissection. Fan et al. (5) showed that white blood cell count predicts 30-day mortality following acute aortic dissection. Bedel et al. and $\mathrm{Oz}$ et al. $(6,7)$ showed that the granulocyte/lymphocyte ratio is an important risk factor for acute aortic dissection at an early stage, and it is also an independent risk factor of early postoperative death, which can be used as a predictor of early postoperative death. In this study, through multivariate regression analysis, the difference of the NLR between the postoperative death and survival groups was statistically significant, with $\mathrm{P}=0.008$, OR: 1.051 (95\% CI: 1.013, 1.091), and the NLR before operation was an independent risk factor for hospital death after ATAAD, which is consistent with the results of previous literature.

When aortic dissection occurs, the intima of the aortic wall tears and the middle layer peels off, forming a false lumen, blood enters the false lumen, activating the coagulation system, thrombosis occurs in the false lumen, leading to activation of fibrinogen precursor, crosslinking of fibrin monomer by activating factor XIII, and hydrolysis of fibrinolytic enzyme to produce a degradation product, namely $\mathrm{D}$-dimer. The increase of $\mathrm{D}$-dimer has high specificity for the diagnosis of aortic dissection. Sbarouni et al. (8) found that the level of D-dimer in acute aortic dissection was significantly higher than that in chronic aortic dissection. It is possible that the proliferation of false lumen endothelial cells in chronic aortic dissection covers the middle layer, such that the coagulation and fibrinolysis system can no longer be activated. The plasma concentration of D-dimer is related to the length of blood vessels involved in dissection, the size of the dissection area and the characteristics of injury. The dissection involves a large area, and the degree of D-dimer and mortality are high. The results of Fan et al. and Wen et al. (9) show that the increase of plasma D-dimer concentration is not only meaningful for the diagnosis of acute aortic dissection but is also important for the prognosis. Itagaki et al. (10) showed that the high concentration of $\mathrm{D}$-dimer before the operation is related to the wide area of dissection and false cavity, which is often accompanied by the decrease of platelet count, long operation time and high probability of blood transfusion, thus increasing the risk of the operation and the risk of death after operation. The results of this study showed that the concentration of D-dimer in the death and survival groups was significantly higher than normal before the operation, and the concentration of $\mathrm{D}$-dimer in the death group was higher than that in the survival group after operation $[\mathrm{P}=0.032$, OR: 1.068 (95\% CI: $1.006,1.134)]$, which is consistent with previous related results. The increase of D-dimer concentration before the operation not only has a high specificity for the diagnosis of acute aortic dissection, but also has high predictive value for the degree of illness and the risk of postoperative death.

After the occurrence of aortic dissection, blood enters the aortic pseudolumen, and coagulation factors come into contact with vascular wall adhesion factors, which stimulates the exogenous coagulation function and consumes a large amount of coagulation substances, resulting in excessive consumption of coagulation substances including platelets. Acute aortic dissection is often accompanied by decreased platelet count and decreased platelet function. On the one hand, pseudoluminal thrombosis promotes excessive platelet consumption, on the other hand, inflammatory reaction can promote platelet activation, and the increase of average platelet volume represents the intensification of platelet activation. Platelet count and function decrease, blood loss increases, and blood product infusion increases, which in turn increases hypoxemia and renal function damage and affects prognosis. Previous studies (11) have shown that patients with acute aortic dissection and aortic aneurysm have lower platelet counts and a higher platelet average volume/platelet count ratio. The related research on the death of dissection shows that the preoperative platelet count is lower and the ratio of platelet average volume to platelet count is higher, which in turn increases the incidence of postoperative complications, increases the postoperative mortality and affects the prognosis (12-14). The results of this study show that there is a significant difference between the preoperative platelet volume in the postoperative death group and the survival group of patients with dissection, the former is higher than the latter, and the preoperative platelet volume is an independent 
risk factor for postoperative death, which is predictive for postoperative death risk.

Cardiac troponin (cTn) is specifically expressed in cardiac myocytes, but not in vascular smooth muscle cells. It is a biological marker of specificity and sensitivity in acute myocardial injury. Related studies have confirmed that cTn is higher than normal in most patients with aortic dissection. On the one hand, the coronary artery is involved in dissection, and the cardiac muscle damage causes troponin to rise; on the other hand, the volume change of dissection leads to the increase of ventricular wall pressure caused by the increase of left ventricular filling pressure, the damage of inflammatory factors, the release of a large amount of catecholamine, oxidative stress and direct mechanical damage of cardiac muscle cells, which leads to damage of cardiac muscle cell integrity and the increase of cTn level. The elevation of cTn in patients with aortic dissection is closely related to the prognosis evaluation. It has been reported in the literature (15) that the mortality rate of patients with acute aortic dissection complicated with elevated $\mathrm{c} T \mathrm{n}$ is four times that of patients with normal cTn, suggesting that high level of $\mathrm{c} T n$ predicts poor prognosis. Clinical detection of hs-CTnT is more sensitive than cTnT. Studies show that the level of hs-CTnT in patients with aortic dissection is higher than that in patients who survive, and that the level of hs-CTnT can independently predict the risk of hospital death in patients with type A AAD. A meta-analysis involving 496 patients $(15,16)$ showed that the risk of hospitalization death of patients with elevated troponin in aortic dissection was significantly higher than that of normal patients [OR: 2.6 (95\% CI: 1.7-4.0)]. In this study, the preoperative hs-CTnT level of patients with dissection was higher than normal, while that of the death group was higher than that of the survival group, with no significant difference $(\mathrm{P}=0.053)$. However, after adjusting for other factors, after multivariate logistic regression analysis, the preoperative hs-CTnT was an independent risk factor for early death after dissection, $\mathrm{P}=0.010$, $\mathrm{OR}$ : 2.023 (95\% CI: 1.183, 3.461). It shows that aortic dissection complicated with myocardial injury, acute change of ventricular volume load, and activation of inflammatory reaction can all increase the risk of postoperative death.

There have been many reports in the literature on studies of renal insufficiency and surgical treatment of aortic dissection, most of which are the results of postoperative renal insufficiency. There is agreement that postoperative renal insufficiency increases all-cause mortality after dissection. Few studies have assessed the influence of preoperative renal function injury on postoperative prognosis.

Fan et al. studied 159 cases of type A aortic dissection. Based on preoperative Cr levels, patients with preoperative Cr greater than $1.5 \mathrm{mg} / \mathrm{dL}$ had postoperative complications and mortality significantly higher than those with preoperative $\mathrm{Cr}$ less than $1.5 \mathrm{mg} / \mathrm{dL}$. These results show that an increase of $\mathrm{Cr}$ before the operation can increase the mortality after operation. The results of this study also confirmed that preoperative $\mathrm{Cr}$ increase is an independent risk factor for postoperative death (17). Wang et al.'s research shows that preoperative renal insufficiency is closely related to the increase of postoperative complications. The postoperative mortality of patients with renal insufficiency is higher than that of those without renal insufficiency, but there is no significant difference between them (18). Similar to the results of this study, the higher the preoperative levels of $\mathrm{Cr}$, the higher the risk of postoperative death, and hence preoperative $\mathrm{Cr}$ is an independent risk factor for postoperative death.

Neurological complications are one of the common complications after aortic dissection. Preoperative cerebrovascular malperfusion, intercalation of superior aortic arch branches, hypotension, old age, time of cardiopulmonary bypass, and long time of selective cerebral perfusion, all of these may be the risk factors of the stroke after surgery. Many studies $(19,20)$ have confirmed that the perioperative mortality rate of patients with organ hypoperfusion is significantly higher. The involvement of supra-arch branch vessels can lead to poor blood supply to nervous system and upper limbs and increase the occurrence of brain complications. The left subclavian artery is an important artery branch of the aortic arch, and if dissected, can increase the occurrence of nervous system ischemia, coma, and stroke, affect the prognosis and increase mortality (21). Authors have drawn different conclusions regarding the prognosis of patients with left subclavian artery involved in dissection. In particular, Hajibandeh et al. (22) showed that occlusion of the left subclavian artery by stent did not increase the risk of perioperative neurological complications. A meta-analysis by Rizvi et al. (23) shows that occlusion of the left subclavian artery can increase the occurrence of cerebral ischemia, cerebral infarction, paraplegia, and limb ischemia and increase the mortality. The results of this study showed that 15 cases $(45.5 \%)$ of the death group were related to cerebral death, and the incidence of stroke was higher than that of the survival group. The dissection involved the left subclavian artery, which was an independent risk factor for postoperative 
death and increased the risk of early postoperative death. The reason led to the result may be related to the influence of dissection on nervous system blood supply, plaque shedding and increase the probability of cerebral ischemia, stroke, and spinal cord ischemia after operation. Aortic dissection involves the iliac artery and femoral artery and its branches, and it may lead to insufficient blood supply to lower limbs, while in severe cases it may lead to limb ischemia, necrosis, and death. Lower extremity arterial hypoperfusion is the most common organ hypoperfusion in ATAAD. It has been reported that $14.5 \%$ of ATAADs are accompanied by lower extremity arterial hypoperfusion (24). The degree of lower limb ischemia is closely related to prognosis. Rutherford et al. (25) divided acute lower limb ischemia into three grades (I, II and III) according to clinical manifestation, outcome, and prognosis. The higher the grade, the worse the prognosis and the higher the mortality. In this study, there were 78 cases $(34.8 \%)$ with iliac artery involvement in the dissection, 55 cases $(28.8 \%)$ in the survival group and 23 cases $(69.7 \%)$ in the death group. There were 10 cases $(4.5 \%)$ with lower limb ischemia before the operation, 7 cases $(3.7 \%)$ in the survival group and 3 cases $(9.1 \%)$ in the death group. There was a significant difference between the two groups. Iliac artery involvement in dissection was an independent risk factor for postoperative death, which is consistent with the results reported in the previous literature. For patients with iliac artery involvement in the dissection before operation, making a reasonable treatment plan, early and effective treatment, and improving limb blood supply can reduce the risk of death caused by limb ischemia.

Preoperative patients with hypoperfusion syndrome, such as intestinal ischemia and necrosis, coma, and acute myocardial infarction, were not included in the research results. Given the preoperative case data and the current medical situation in China, patients with dissection first went to primary medical units after onset, and some patients with organ hypoperfusion died because they could not be referred to hospitals with surgical ability in time. Even so, our research results still show that preoperative iliac artery and subclavian artery involvement in dissections is still an important predictive risk factor for postoperative death. Therefore, for patients who have no obvious ischemia and poor perfusion before operation, but whose branch arteries are involved in the dissection as evidenced by enhanced CT, we should attach great importance to these manifestations and commence surgical treatment as soon as possible to avoid further aggravation of ischemic damage, thus reducing the risk of postoperative death.
Shortcomings of this study are: it is a single-center retrospective study, the number of selected cases is small, the number of cases with severe hypoperfusion complications before operation is small, and there is a bias in case selection. The end point of this study is hospital death, and there is a lack of medium- and long-term followup results, and as such it is inevitable that there are some shortcomings regarding the accuracy of the outcome. In the follow-up work, long-term outcomes, adverse events and reoperation will be added. In this study, the prediction model is only tested internally, and it needs to be further verified externally to comprehensively evaluate the prediction efficiency of the model.

To conclude, this study is based on preoperative laboratory examination, clinical imaging data, and early postoperative treatment outcome of patients with aortic dissection. This clinical information can be obtained easily. This death risk model is simple and easy to follow and can be used to evaluate the condition and treatment risk, predict the probability of death in patients with aortic dissection, guide the formulation and implementation of reasonable and effective treatment programs, improve the prognosis and survival rate and reduce the mortality rate.

\section{Acknowledgments}

Funding: This work was supported by the Tianjin Commission of Science and Technology (No. 18ZXDBSY00160) and the Tianjin Municipal Bureau of Health (No. ZC20087).

\section{Footnote}

Reporting Checklist: The authors have completed the STARD reporting checklist. Available at https://dx.doi. org/10.21037/atm-21-4063

Data Sharing Statement: Available at https://dx.doi. org/10.21037/atm-21-4063

Conflicts of Interest: All authors have completed the ICMJE uniform disclosure form (available at https://dx.doi. org/10.21037/atm-21-4063). The authors have no conflicts of interest to declare.

Ethical Statement: The authors are accountable for all aspects of the work in ensuring that questions related to the accuracy or integrity of any part of the work are 
appropriately investigated and resolved. All procedures performed in this study involving human participants were in accordance with the Declaration of Helsinki (as revised in 2013). This study is a single-center retrospective study, which has been approved by the Ethics Committee of Tianjin Chest Hospital. Individual consent for this retrospective analysis was waived.

Open Access Statement: This is an Open Access article distributed in accordance with the Creative Commons Attribution-NonCommercial-NoDerivs 4.0 International License (CC BY-NC-ND 4.0), which permits the noncommercial replication and distribution of the article with the strict proviso that no changes or edits are made and the original work is properly cited (including links to both the formal publication through the relevant DOI and the license). See: https://creativecommons.org/licenses/by-nc-nd/4.0/.

\section{References}

1. Pape LA, Awais M, Woznicki EM, et al. Presentation, diagnosis, and outcomes of acute aortic dissection: 17-year trends from the International Registry of Acute Aortic Dissection. J Am Coll Cardiol 2015;66:350-8.

2. Ma WG, Zhu JM, Zheng J, et al. Sun's procedure for complex aortic arch repair: total arch replacement using a tetrafurcate graft with stented elephant trunk implantation. Ann Cardiothorac Surg 2013;2:642-8.

3. Czerny M, Schoenhoff F, Etz C, et al. The impact of preoperative malperfusion on outcome in acute type A aortic dissection: results from the GERAADA Registry. J Am Coll Cardiol 2015;65:2628-35.

4. Ghoreishi M, Wise ES, Croal-Abrahams L, et al. A novel risk score predicts operative mortality after acute type A aortic dissection repair. Ann Thorac Surg 2018;106:1759-66.

5. Fan X, Huang B, Lu H, et al. Impact of admission white blood cell count on short- and long-term mortality in patients with type A acute aortic dissection: an observational study. Medicine (Baltimore) 2015;94:e1761.

6. Bedel C, Selvi F. Association of platelet to lymphocyte and neutrophil to lymphocyte ratios with in-hospital mortality in patients with type A acute aortic dissection. Braz J Cardiovasc Surg 2019;34:694-8.

7. Oz K, Iyigun T, Karaman Z, et al. Prognostic value of neutrophil to lymphocyte ratio and risk factors for mortality in patients with stanford type A aortic dissection. Heart Surg Forum 2017;20:E119-23.
8. Sbarouni E, Georgiadou P, Marathias A, et al. D-dimer and BNP levels in acute aortic dissection. Int J Cardiol 2007;122:170-2.

9. Wen D, Du X, Dong JZ, et al. Value of D-dimer and C reactive protein in predicting inhospital death in acute aortic dissection. Heart 2013;99:1192-7.

10. Itagaki R, Kimura N, Mieno M, et al. Characteristics and treatment outcomes of acute type A aortic dissection with elevated D-dimer concentration. J Am Heart Assoc 2018;7:009144.

11. Chen Y, Lin Y, Zhang H, et al. Relationship of platelet counts and inflammatory markers to 30-day mortality risk in patients with acute type A aortic dissection. Biomed Res Int 2020;2020:1057496.

12. Sbarouni E, Georgiadou P, Analitis A, et al. Significant changes in platelet count, volume and size in acute aortic dissection. Int J Cardiol 2013;168:4349-50.

13. Kamisli O, Kamisli S, Gonullu S, et al. Increased mean platelet volume in cervicocephalic artery dissections. Ir J Med Sci 2012;181:561-5.

14. Li DZ, Chen QJ, Sun HP, et al. Mean platelet volume to platelet count ratio predicts in-hospital complications and long-term mortality in type A acute aortic dissection. Blood Coagul Fibrinolysis 2016;27:653-9.

15. Hiratzka LF, Bakris GL, Beckman JA, et al. 2010 ACCF/AHA/AATS/ACR/ASA/SCA/SCAI/SIR/STS/ SVM guidelines for the diagnosis and management of patients with thoracic aortic disease: a report of the American College of Cardiology Foundation/American Heart Association Task Force on Practice Guidelines, American Association for Thoracic Surgery, American College of Radiology, American Stroke Association, Society of Cardiovascular Anesthesiologists, Society for Cardiovascular Angiography and Interventions, Society of Interventional Radiology, Society of Thoracic Surgeons, and Society for Vascular Medicine. Circulation 2010;121:e266-369. Erratum in: Circulation 2010;122:e410.

16. Vrsalovic M. Prognostic effect of cardiac troponin elevation in acute aortic dissection: A meta-analysis. Int J Cardiol 2016;214:277-8.

17. Fan PY, Chen CY, Lee CC, et al. Impact of renal dysfunction on surgical outcomes in patients with aortic dissection. Medicine (Baltimore) 2019;98:e15453.

18. Wang X, Ren HM, Hu CY, et al. Predictors and inhospital outcomes of preoperative acute kidney injury in patients with type A acute aortic dissection. J Geriatr Cardiol 2016;13:679-84. 
19. Augoustides JG, Szeto WY, Desai ND, et al. Classification of acute type A dissection: focus on clinical presentation and extent. Eur J Cardiothorac Surg 2011;39:519-22.

20. Caus T, Frapier JM, Giorgi R, et al. Clinical outcome after repair of acute type A dissection in patients over 70 yearsold. Eur J Cardiothorac Surg 2002;22:211-7.

21. Conzelmann LO, Weigang E, Mehlhorn U, et al. Mortality in patients with acute aortic dissection type A: analysis of pre- and intraoperative risk factors from the German Registry for Acute Aortic Dissection Type A (GERAADA). Eur J Cardiothorac Surg 2016;49:e44-52.

22. Hajibandeh S, Hajibandeh S, Antoniou SA, et al. Metaanalysis of left subclavian artery coverage with and without revascularization in thoracic endovascular aortic repair. J
Endovasc Ther 2016;23:634-41.

23. Rizvi AZ, Murad MH, Fairman RM, et al. The effect of left subclavian artery coverage on morbidity and mortality in patients undergoing endovascular thoracic aortic interventions: a systematic review and meta-analysis. J Vasc Surg 2009;50:1159-69.

24. Immer FF, Grobéty V, Lauten A, et al. Does malperfusion syndrome affect early and mid-term outcome in patients suffering from acute type A aortic dissection? Interact Cardiovasc Thorac Surg 2006;5:187-90.

25. Rutherford RB, Baker JD, Ernst C, et al. Recommended standards for reports dealing with lower extremity ischemia: revised version. J Vasc Surg 1997;26:517-38.

(English Language Editor: B. Meiser)
Cite this article as: Zhang Y, Chen T, Chen Q, Min H, Nan J, Guo Z. Development and evaluation of an early death risk prediction model after acute type A aortic dissection. Ann Transl Med 2021;9(18):1442. doi: 10.21037/atm-21-4063 